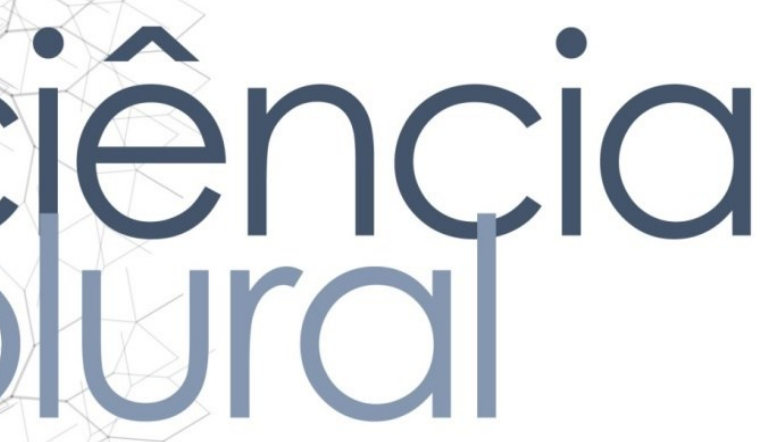

\title{
AVALIAÇÃO DO ATRIBUTO COORDENAÇÃO DO CUIDADO EM SERVIÇOS DE PUERICULTURA NA ATENÇÃO PRIMÁRIA À SAÚDE
}

Evaluation of the attribute coordination of care in childcare services in Primary Health Care

Tainara Lôrena dos Santos Ferreira • Acadêmica do Curso de Enfermagem na Faculdade de Ciências da Saúde do Trairi (FACISA)/Universidade Federal do Rio Grande do Norte (UFRN). Bolsista de Iniciação Científica PIBIC-CNPq/UFRN. E-mail: tainara_lorena@hotmail.com

Ana Mayara Gomes de Souza - Acadêmica do Curso de Enfermagem na FACISA/UFRN. E-mail: ana.mayara@yahoo.com.br

Joycimara da Silva Sales de Medeiros - Acadêmica do Curso de Enfermagem na FACISA/UFRN. E-mail: joycimara93@hotmail.com

Fábia Barbosa de Andrade • Enfermeira. Doutora em Ciências da Saúde. Professora Adjunto III da UFRN. E-mail: fabiabarbosabr@yahoo.com.br

Autora responsável pela correspondência:

Tainara Lôrena dos Santos Ferreira. E-mail: tainara_lorena@hotmail.com 


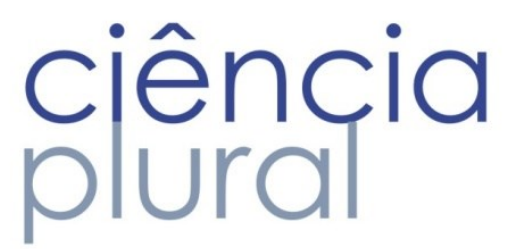

\section{RESUMO}

Introdução: A coordenação do cuidado trata-se de um atributo essencial dos serviços que tem por finalidade, organizar os atendimentos aos usuários, possibilitar acesso livre as informações e garantir resolutividade da assistência em saúde. Objetivo: Avaliar a presença e extensão do atributo coordenação do cuidado em serviços de Puericultura. Métodos: Trata-se de um estudo avaliativo, quantitativo, de caráter descritivo, com amostra composta por 186 pais/responsáveis de crianças de 0 à 2 anos de idade que utilizam os serviços da Atenção Primária à Saúde, utilizando um questionário validado no Brasil denominado PCA Tools, versão para crianças. Resultados: No que se refere à integração de cuidados esta se apresentou com resolutividade de queixas de $89,2 \%$, uma vez que apenas 10,8\% foram consultados por especialistas, porém na ótica dos usuários, $7,5 \%$ afirmam ainda que os profissionais se mostraram interessados na qualidade do cuidado prestada no serviço especializado. Já no que se refere ao sistema de informações, 99,5\% afirmaram disponibilidade dos registros e boletins de atendimentos durante a consulta. Conclusão: $O$ estudo permitiu identificar potencialidades e avanços em relação ao atributo estudado. As fragilidades ainda existentes ajudam na reflexão e motivação dos profissionais, em conseguir uma totalidade na assistência ao cuidado infantil.

Palavras-chave: Atenção Primária à Saúde, Saúde da Criança, Avaliação de Serviços de Saúde.

\section{ABSTRACT}

Introduction: The coordination of care is an essential attribute of the services that have as purpose, organize the services to the users, enable free access to information and guarantee the resolution of health care. Objective: To evaluate the presence and extension of the coordination attribute of care in childcare services. Methods: This is an evaluative, quantitative, descriptive study with a sample composed of 186 parents/guardians of children from 0 to 2 years of age who use Primary Health Care services, using a questionnaire validated in Brazil PCA Tools, children's version. Results: Regarding the integration of care, this was presented with a complaints resolution of $89.2 \%$, since only $10.8 \%$ were consulted by specialists, but from the users' perspective, $7.5 \%$ Professionals were interested in the quality of care provided in the specialized service. Regarding the information system, $99.5 \%$ reported availability of records and reports during the consultation. Conclusion: The study allowed to identify potentialities and advances in relation to the attribute studied. The still existing fragilities help in the reflection and motivation of the professionals to achieve a totality in the assistance to the child care.

Key words: Primary Health Care, Child Health, Health Services Evaluation. 


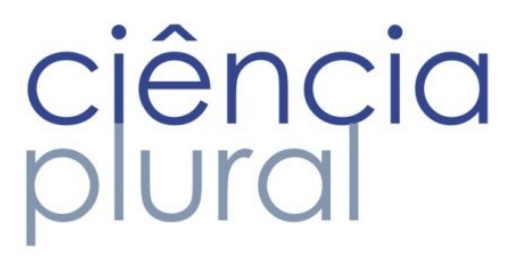

\section{Introdução}

A Atenção Primária à Saúde (APS) tem se apresentado com positividade no contexto da melhoria da assistência por apresentar-se como centro de comunicação da Rede de Atenção à Saúde (RAS), sendo vista como o primeiro nível de atenção e porta de entrada preferencial para os outros níveis do Sistema Único de Saúde (SUS). No âmbito da saúde da criança, o programa de puericultura se propõe ao acompanhamento da saúde infantil, a fim de garantir um crescimento saudável, sendo responsável pela promoção, prevenção e recuperação dos indivíduos nessa fase da vida, tornando-se eixo fundamental do SUS por proporcionar acesso de qualidade às ações de saúde.

Historicamente, emerge a problemática da segmentação e fragmentação na atenção a saúde, e esta possui estreita relação com a qualidade do serviço, uma vez que reflete na continuidade do cuidado, integralidade e perda de eficiência ${ }^{1}$. Para que a atenção em saúde seja ofertada com qualidade no âmbito da APS são propostos alguns atributos, tais como: o primeiro contato, a longitudinalidade, a integralidade, a coordenação, a focalização na família e a orientação comunitária².

A integração da saúde, com constituição de redes regionalizadas e integradas de atenção tornase, portanto, condição indispensável para a qualificação e a continuidade do cuidado à saúde, uma vez que se apresenta como estratégias para a superação de lacunas assistenciais, racionalização e otimização dos recursos assistenciais disponíveis ${ }^{3}$. Deste modo, a coordenação é a articulação entre os diversos serviços e ações relacionadas à saúde possuindo grande relevância para os demais atributos, necessitando estarem sincronizadas e contínuas, independentemente do local em que ocorrem ou do tipo de atendimento profissional prestado em qualquer nível de complexidade 4 .

A fragmentação da rede assistencial, ausência de comunicação entre prestadores, assim como, os problemas vinculados à coordenação do cuidado, ainda são temas bastante recorrentes no que diz respeito à organização do sistema de saúde ${ }^{5}$. Dessa forma devem ser considerados relevantes e priorizados em todas as instâncias gerenciais da saúde, na garantia de atribuir ao setor saúde maior integralidade e universalidade da assistência.

Segundo autores ${ }^{4,5,6}$, o desenvolvimento e implantação de novas tecnologias de informação atuam como aliados para a continuidade da informação, uma vez que tais instrumentos agilizam as etapas de registro, processamento e disponibilização das informações em saúde, orientando a conduta terapêutica e favorecendo a avaliação. No entanto, critérios como suficiência e confiabilidade são de responsabilidade dos profissionais que realizam os atendimentos, posto que a fonte do dado seja quase sempre o paciente em seu contato com a equipe de saúde. 


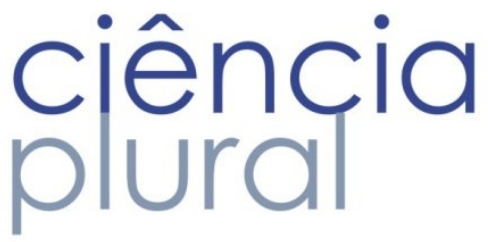

Deste modo, observa-se que a modernização e aplicabilidade de técnicas e registros, concorrem para o aperfeiçoamento da assistência, com vistas a tornar o serviço menos fragmentado e mais completo, alem de facilitar 0 trabalho do profissional e a melhor interação com o paciente, cumprindo assim o seu papel de prover saúde. Ressalta-se ainda segundo esse estudo ${ }^{7}$, que a APS passa a ser uma estratégia de organização e reorganização dos sistemas de saúde, cujo modelo sofreu mudanças que enfatizam a prática clínico-assistencial exercida pela equipe de saúde, funcionando como reordenadora dos serviços, orientada por atributos essenciais.

Destarte, por entender a importância da presença dos atributos essenciais e derivados, e por serem indispensáveis às práticas de registros de saúde na garantia da qualidade do acesso as informações disponíveis no serviço, este estudo tem por objetivo avaliar a presença e extensão do atributo coordenação do cuidado em serviços de Puericultura na Atenção Primária à Saúde, a partir da percepção de pais/responsáveis por crianças menores de dois anos cadastradas no serviço.

\section{Metodologia}

Trata-se de um estudo exploratório descritivo, avaliativo e com abordagem quantitativa realizado na cidade de Santa Cruz, Rio Grande do Norte, Brasil, realizado com pais/responsáveis por crianças menores de dois anos, atendidas em Unidades Básicas de Saúde (UBS). 0 presente estudo trata-se da primeira etapa de uma pesquisa maior intitulada "Construção e validação de escala nos serviços de Puericultura: um estudo de avaliação na Atenção Primária à Saúde".

A escolha da amostra se deu de modo aleatório, com base no cadastro das Unidades Saúde da Família (USF) e com respeito à proporcionalidade dos usuários cadastrados, sendo adotado erro permitido de $5 \%$ e grau de confiança de $95 \%$, além de um poder de $80 \%$, mediante assinatura das mães de crianças, resultando em uma amostra de 186 crianças, acrescido já às perdas do estudo.

Para a coleta de dados foi utilizado o instrumento validado pelo Ministério da Saúde intitulado Primary Care Assessment Tools (PCA Tools) ${ }^{8}$ versão para crianças, a partir dos atributos do sistema de serviços de saúde, que são: acesso de primeiro contato, integralidade, longitudinalidade, coordenação, orientação familiar e comunitária e competência cultural2.

Os dados coletados foram armazenados e analisados no Statistical Package for the Social Sciences (SPSS), versão 22.0, com número de série: 10101141047. Utilizou-se neste estudo as variáveis para caracterizar a população e que constituem o atributo "coordenação do cuidado". Para tanto, realizou- 


\section{ciência \\ plural}

se análise descritiva a partir de frequências absoluto-relativas, medidas de tendência central e de dispersão. Para verificar a consistência interna e a homogeneidade das variâncias, realizou-se ainda o alfa de Cronbach e teste de Anova Cochran.

Este estudo foi realizado após apreciação do Comitê de Ética em Pesquisa da Faculdade de Ciências da Saúde do Trairi/Universidade Federal do Rio Grande do Norte, segundo o que preconiza a Resolução 466/2012 do Conselho Nacional de Saúde ${ }^{9}$ sobre a pesquisa com seres humanos, sob número de parecer 348.896 .

\section{Resultados}

O presente estudo buscou avaliar o atributo coordenação do serviço de puericultura oferecido na APS, tendo como base os princípios do SUS, considerando as necessidades de acordo com a demanda e com os níveis de complexidade dos serviços ofertados. Para os autores ${ }^{1,2}$, estudos da coordenação do cuidado possibilitam a avaliação do desempenho de sistemas orientados por uma política de APS.

Tabela 1: Distribuição dos resultados da validação de construto do atributo Coordenação (Integração de Cuidados) na Atenção Primária à Saúde. Santa Cruz-RN, Brasil, 2016.

\begin{tabular}{|c|c|c|c|c|}
\hline Variáveis & $\begin{array}{l}\text { Com certeza, } \\
\text { não }\end{array}$ & $\begin{array}{l}\text { Provavelmente, } \\
\text { não }\end{array}$ & $\begin{array}{l}\text { Provavelmente, } \\
\text { sim }\end{array}$ & $\begin{array}{l}\text { Com certeza, } \\
\text { sim }\end{array}$ \\
\hline $\begin{array}{l}\text { Solicitação de consulta } \\
\text { ao especialista pela } \\
\text { APS }\end{array}$ & $\begin{array}{c}5,9 \% \\
(n=11)\end{array}$ & - & - & $\begin{array}{c}4,8 \% \\
(n=09)\end{array}$ \\
\hline $\begin{array}{l}\text { O profissional da APS } \\
\text { que a acompanha sabe } \\
\text { da consulta ao } \\
\text { especialista }\end{array}$ & $\begin{array}{l}1,6 \% \\
(n=03)\end{array}$ & - & - & $\begin{array}{l}9,1 \% \\
(n=17)\end{array}$ \\
\hline $\begin{array}{l}\text { O profissional da APS } \\
\text { sabe dos resultados } \\
\text { desta consulta }\end{array}$ & $\begin{array}{l}3,8 \% \\
(n=07)\end{array}$ & - & - & $\begin{array}{c}7,0 \% \\
(n=13)\end{array}$ \\
\hline $\begin{array}{l}\text { O profissional da APS } \\
\text { conversou sobre a } \\
\text { consulta após a mesma }\end{array}$ & $\begin{array}{l}3,8 \% \\
(n=07)\end{array}$ & - & - & $\begin{array}{l}7,0 \% \\
(n=13)\end{array}$ \\
\hline $\begin{array}{l}\text { O profissional da APS } \\
\text { pareceu interessado na } \\
\text { qualidade do cuidado } \\
\text { prestado na consulta }\end{array}$ & $\begin{array}{l}1,6 \% \\
(n=03)\end{array}$ & - & $\begin{array}{l}1,6 \% \\
(n=03)\end{array}$ & $\begin{array}{l}7,5 \% \\
(n=14)\end{array}$ \\
\hline
\end{tabular}

Para tanto, foram entrevistados 186 pais/responsáveis de crianças com idade inferior a dois anos, das quais 106 (57,0\%) crianças eram do sexo masculino e 80 (43,0\%) eram do sexo feminino. A média de 


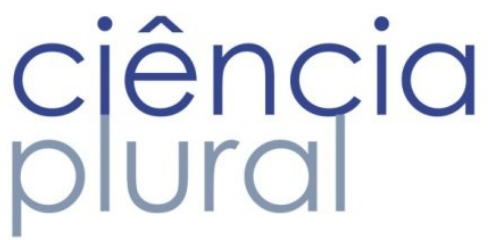

idade das crianças em meses foi de 8,21; e obteve-se mediana de 7,00 e o desvio padrão de 6,31. Quanto à escolaridade da mãe, a média foi em torno de 10,46 e mediana 9,00 . Apenas $20(10,8 \%)$ afirmaram que a criança foi à consulta de especialista/serviço especializado no período de seu acompanhamento pela APS.

Quando questionados quanto ao atributo Coordenação (Integração de Cuidados), 20 (10,8\%) foram consultar qualquer tipo de especialista ou serviço especializado no período em que ela está em acompanhamento na UBS; 11 (5,9\%) não foram encaminhados pelo profissional que acompanha a criança; 17 (9,1\%) afirmam que o profissional tem conhecimento sobre a realização da consulta e 13 (7,0\%) saber os resultados, e ter conversado sobre a consulta após a mesma. Na ótica dos usuários, 14 (7,5\%) afirmaram que o profissional se mostrou interessado na qualidade do cuidado prestada no serviço especializado.

Tabela 02: Distribuição dos resultados da validação de construto do atributo Coordenação (Sistema de Informações) da Atenção Primária à Saúde. Santa Cruz-RN, Brasil, 2016.

\begin{tabular}{|c|c|c|c|c|}
\hline Variáveis & $\begin{array}{l}\text { Com certeza, } \\
\text { não }\end{array}$ & $\begin{array}{l}\text { Provavelmente, } \\
\text { não }\end{array}$ & $\begin{array}{l}\text { Provavelmente, } \\
\text { sim }\end{array}$ & $\begin{array}{l}\text { Com certeza, } \\
\text { sim }\end{array}$ \\
\hline $\begin{array}{l}\text { Registros de saúde da } \\
\text { criança disponíveis no } \\
\text { serviço }\end{array}$ & $\begin{array}{c}1,1 \% \\
(n=02)\end{array}$ & - & - & $\begin{array}{c}98,9 \% \\
(n=184)\end{array}$ \\
\hline $\begin{array}{l}\text { Prontuário da criança } \\
\text { disponível na consulta }\end{array}$ & $\begin{array}{l}0,5 \% \\
(n=01)\end{array}$ & - & - & $\begin{array}{l}99,5 \% \\
(n=185)\end{array}$ \\
\hline $\begin{array}{lr}\text { Possibilidade } & \text { de } \\
\text { ler/consultar } & 0 \\
\text { prontuário da } & \text { criança } \\
\text { caso queira } & \end{array}$ & $\begin{array}{c}7,5 \% \\
(n=14)\end{array}$ & $\begin{array}{l}8,1 \% \\
(n=15)\end{array}$ & $\begin{array}{l}15,6 \% \\
(n=29)\end{array}$ & $\begin{array}{l}51,6 \% \\
(n=96)\end{array}$ \\
\hline
\end{tabular}

Concernente ao atributo Coordenação (Sistema de Informações), 184 (98,9\%) afirmou que os registros de saúde e boletins de atendimentos passados da criança encontram-se disponíveis no serviço, 185 (99,5\%) afirma que o prontuário da criança está sempre disponível na consulta; 96 (51,6\%) afirma ter a possibilidade de ler/consultar o prontuário caso queira, seguido por 29 (15,6\%) que verbalizam provavelmente sim.

Os dados revelaram um alfa de Cronbach de 0,71, fato que mostra a existência de validade interna e dados fidedignos no constructo. Quanto ao teste de Anova Cochran, foi verificado significância através do $p<0,01$, revelando a homogeneidade das variâncias. Isso mostra que a escala de avaliação se encontra viável e fidedigna para ser usada na rede da APS, como meio de avaliar a qualidade da atenção prestada à população infantil. 


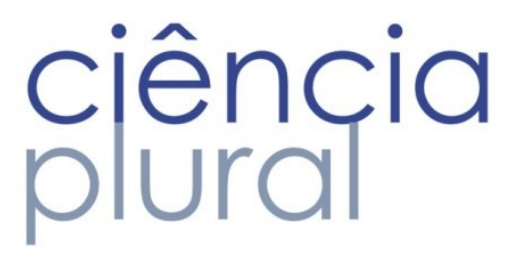

\section{Discussão}

Observaram-se a partir dos dados que as ações concernentes ao atributo coordenação têm sido propostas na perspectiva de pais e responsáveis por crianças menores de dois anos através do serviço de puericultura. Considerando o papel estratégico da APS enquanto ordenadora do sistema de saúde, reconhece-se então a necessidade de avaliar como está estruturada a coordenação ${ }^{10}$. Assim para fins de melhor análise e compreensão dos resultados, as ações deste atributo foram divididas em "integração de cuidados" e "sistema de informações".

No que se refere ao atributo Coordenação (Integração de Cuidados), os resultados da tabela 1 sugerem a resolutividade de queixas na grande maioria da amostra, uma vez que apenas 10,8\% foram consultados por especialistas. Destes, observa-se que embora não tenha sido em sua totalidade encaminhados pelos serviços que os acompanhavam, evidencia-se que ainda sim, este atributo tem sido considerado relevante para a manutenção da continuidade da assistência em UBS, por ter o conhecimento sobre a realização da consulta e estar ciente dos resultados e ainda ter conversado sobre a consulta após a mesma. Observou-se ainda que o profissional se mostrou interessado na qualidade do cuidado prestada no serviço especializado, na ótica dos usuários.

Na situação em que se encontra à saúde do país, a valorização e defesa da concepção abrangente da APS parecem ser promissoras as iniciativas pró-coordenação, pois a coordenação assistencial se apresenta como atributo organizacional que se traduz sistematicamente na percepção de continuidade dos cuidados aos usuários ${ }^{5}$. Para, além disso, a integração das redes do cuidado torna abrangente e seletivo os níveis assistenciais, tornando-os de alta qualidade e efetividade, e contribuindo para que os profissionais atuem de forma multiprofissional e articulada tendo acesso à devolução situacional das condutas adotadas em todos os meios para onde o usuário é encaminhado.

Entretanto, apesar das constantes implementações e aplicabilidade de técnicas e registros nos serviços de saúde, os serviços públicos no Brasil ainda são caracterizados pela fragmentação. Conceitualmente ${ }^{11}$, os sistemas fragmentados de atenção à saúde são aqueles que se organizam através de um conjunto de pontos de atenção à saúde isolados e incomunicados uns dos outros e que, por consequência, são incapazes de prestar uma atenção contínua à população. A ausência da contrareferência sugere necessidade de maior integração entre profissionais da atenção básica e especializada 5 .

A busca por alcançar os objetivos essenciais da assistência no SUS sejam eles, à universalização, equidade e integralidade, na garantia de proporcionar aos usuários um acolhimento articulado, de qualidade, baseando-se nos critérios socioeconômicos e culturais, ainda configura-se como grandes 


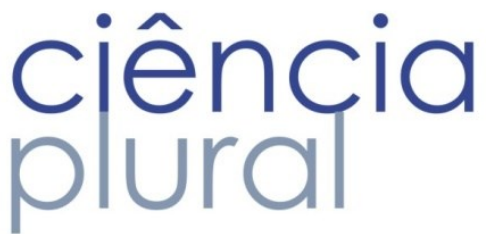

esforços das esferas governamentais, pois é presente nos modelos assistenciais a tendência de fragmentação da clínica sejam internamente ou externamente nos diversos níveis de hierarquização ${ }^{7}$.

A partir dessa fragilidade encontrada, referente ao princípio de integração dos cuidados, pode-se afirmar que, este processo assistencial é fundamental para garantir uma oferta abrangente de serviços, para coordenar as diversas ações requeridas e ainda resolver as necessidades menos frequentes e mais complexas $^{7}$. Isso propõe que os serviços de saúde brasileiros sejam desafiados a darem respostas contínuas e integradas considerando a fragmentação do sistema de saúde.

Entende-se que integração, coordenação e continuidade são inter-relacionadas e é no período da infância em que são desenvolvidas as maiores potencialidades e também são identificados os casos de morbimortalidade, por se desenvolver grande parte das potencialidades humanas que são responsáveis por graves consequências para os indivíduos e comunidades ${ }^{12}$. Assim, uma vez identificados esses distúrbios em consultas resolutivas de puericultura nos serviços especializados, a coordenação torna-se eficaz na integração de cuidados contínuos, sendo estes bem executados na existência de uma rede multiprofissional que priorize a continuidade da contra referência em todas as instâncias e complexidades.

Concernente aos dados apresentados ao atributo Coordenação (Sistema de informações), estes nos revelam quanto a importância do armazenamento e livre acesso às informações, que os registros de saúde e boletins de atendimentos passados da criança encontram-se disponíveis no serviço, com índice de 185 (99,5\%) afirmando tal disponibilidade durante a consulta. Seguindo a compreensão, os dados mostram ainda que na ótica de pais/responsáveis existe a possibilidade de ler/consultar o prontuário da criança, caso queira.

Tais dados permitem observar que uma vez que esses prontuários apresentam-se acessíveis a comunidade, o apoio para melhoria da continuidade do cuidado se torna eficaz e perdurável, por ser um meio do sistema ser considerado efetivo no que se diz respeito à transferência de informação. Por consequência, este necessita que os dados contidos sejam fidedignos e pertinentes, melhorando a compreensão para com o paciente e suas necessidades. Já os valores negativos obtidos nas demais interrogativas nos permite considerar que, esses valores se manifestam devido ao desconhecimento, associado ao desinteresse ou insciência dos usuários à possibilidade de ter a obtenção das informações, dificultando assim a propagação da mesma.

Para alguns autores ${ }^{9,10}$, a utilização do prontuário familiar auxilia na consolidação de vários objetivos, possibilitando o registro das informações em saúde de todos os membros da família, constituindo um importante instrumento para a integração da equipe. A alta disponibilidade dos prontuários de saúde seria adequada a melhoria das informações descritas no mesmo, pois muitas informações ficam restritas 


\section{ciência \\ plural}

à pessoa que escreveu, dificultando a continuidade do atendimento ao paciente, assim como sua compreensão diante dos dados ali contidos ${ }^{4}$.

Nesta perspectiva, ressalta-se que embora haja muitos desafios postos quanto a prestação do cuidado em saúde pautado nos atributos propostos, houve grandes avanços. Destarte a importância percebida na APS como fundamental para o funcionamento das redes de atenção de modo efetivo e resolutivo, como observado na perspectiva de usuários, ressalta-se a importância de estudos de avaliação na ótica de usuários, a fim de identificar potencialidades e fragilidades da assistência a saúde e de conhecer as situações que permeiam a vida dos usuários.

\section{Conclusões}

No cenário atual, ganha destaque a discussão acerca do atributo "coordenação do cuidado", visto que a fragmentação da assistência quando se mostra de modo isolado e incomunicável caracteriza-se pela descontinuidade do cuidado. Observou-se que uma pequena parcela da amostra refere-se à criança ter realizado consulta com especialista no período que esta sendo acompanhado nas consultas de C e D. Tal fato sugere que a maioria da amostra conseguiu a resolutividade através das ações do serviço, no entanto, merece destacar que a parcela que referiu a consulta com o especialista afirma que esta não foi encaminhada pelo serviço que a acompanha.

Percebeu-se nesta pesquisa o interesse dos profissionais em buscar informações sobre a consulta com o especialista no acompanhamento da criança, fato que destaca o comprometimento dos profissionais da rede de APS, para proporcionar um atendimento à saúde com qualidade, evidenciado pela afirmação "com certeza, sim" quando questionados. Além disso, destacou-se o sistema de informações efetivo, através dos registros de saúde e prontuários disponíveis na consulta, bem como acessíveis aos pais/responsáveis da criança.

O estudo permitiu identificar potencialidades e avanços em relação ao atributo "coordenação". As fragilidades ainda existentes ajudam na reflexão e motivação dos profissionais em conseguir uma totalidade na assistência ao cuidado infantil. Finalmente, apesar das limitações no que diz respeito a ser um estudo local, o mesmo pretende contribuir estimulando à elaboração de novas estratégias de melhorias na atuação e acompanhamento da saúde da criança, incentivando o fortalecimento da humanização e crescimento de melhorias na saúde desses pacientes.

\section{Referências}




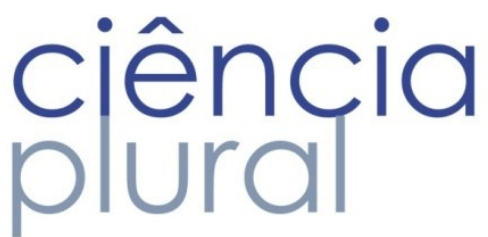

1. Conill EM, Fausto MCR, Giovanella L. Contribuições da análise comparada para um marco abrangente na avaliação de sistemas orientados pela atenção primária na América Latina. Rev Bras Saúde Matern Infant. 2010 nov; 10(Supl. 1): S15-S27.

2. Starfield B. Atenção primária: equilíbrio entre necessidades de saúde, serviços e tecnologia. Brasília: Ministério da Saúde, 2002.

3. Silva SF. Organização de redes regionalizadas e integradas de atenção à saúde: desafios do Sistema Único de Saúde (Brasil). Ciênc Saúde Coletiva. 2011; 16(6): 2753-62.

4. Souza GT, Alves BA; Tacla MTGM, Collet N, Toso BRGO. Avaliação do princípio da coordenação na atenção primária à saúde da criança em Londrina-PR. Semina Cienc. Biol. Saude. 2015 jan.jjun; 36(1): 3946.

5. Almeida PF, Giovanella L, Mendonça MHM, Escorel S. Desafios à coordenação dos cuidados em saúde: estratégias de integração entre níveis assistenciais em grandes centros urbanos. Cad. Saúde Pública. 2010 fev; 26(2): 286-298.

6. Cunha EM, Giovanella L. Longitudinalidade/continuidade do cuidado: identificando dimensões e variáveis para a avaliação da Atenção Primária no contexto do sistema público de saúde brasileiro. Ciênc. Saúde Coletiva. 2011; 16(Supl. 1): 1029-1042.

7. Oliveira MAC, Pereira IC. Atributos essenciais da Atenção Primária e a Estratégia Saúde da Família. Rev. Bras. Enferm. 2013; 66(esp): 158-64.

8. Brasil. Ministério da Saúde. Secretaria de Atenção em Saúde. Departamento de Atenção Básica. Manual do instrumento de avaliação da atenção primária à saúde: Primary Care Assessment Tool PCAtool -Brasil. Brasília (DF): Ministério da Saúde, 2010.

9. Brasil. Ministério da Saúde. Resolução $n^{0}$ 466, de 12 de dezembro de 2012. Diretrizes e normas regulamentadoras de pesquisas envolvendo seres humanos. Ministério da Saúde. Brasília: Ministério da Saúde, 2012.

10. Carneiro MSM, Melo DMS, Gomes JM, Pinto FJM, Silva MGC. Avaliação do atributo coordenação da Atenção Primária à Saúde: aplicação do PCATool a profissionais e usuários. Saúde Debate. 2014 out; 38(esp): 279-295.

11. Mendes EV. As redes de atenção à saúde. Ciênc. Saúde Coletiva. 2010 Aug; 15(5): 2297-2305.

12. Vieira VCL, Fernandes CA, Demitto MO, Bercini LO, Scochi MJ, Marcon SS. Puericultura na Atenção Primária À Saúde: Atuação do Enfermeiro. Cogitare Enferm. 2012 jan/mar; 17(1): 119-25. 